UCRL-ID-131728

\title{
Code Development for Opacity Database
}

\author{
Balazs Rozsnyai
}

July 1, 1998

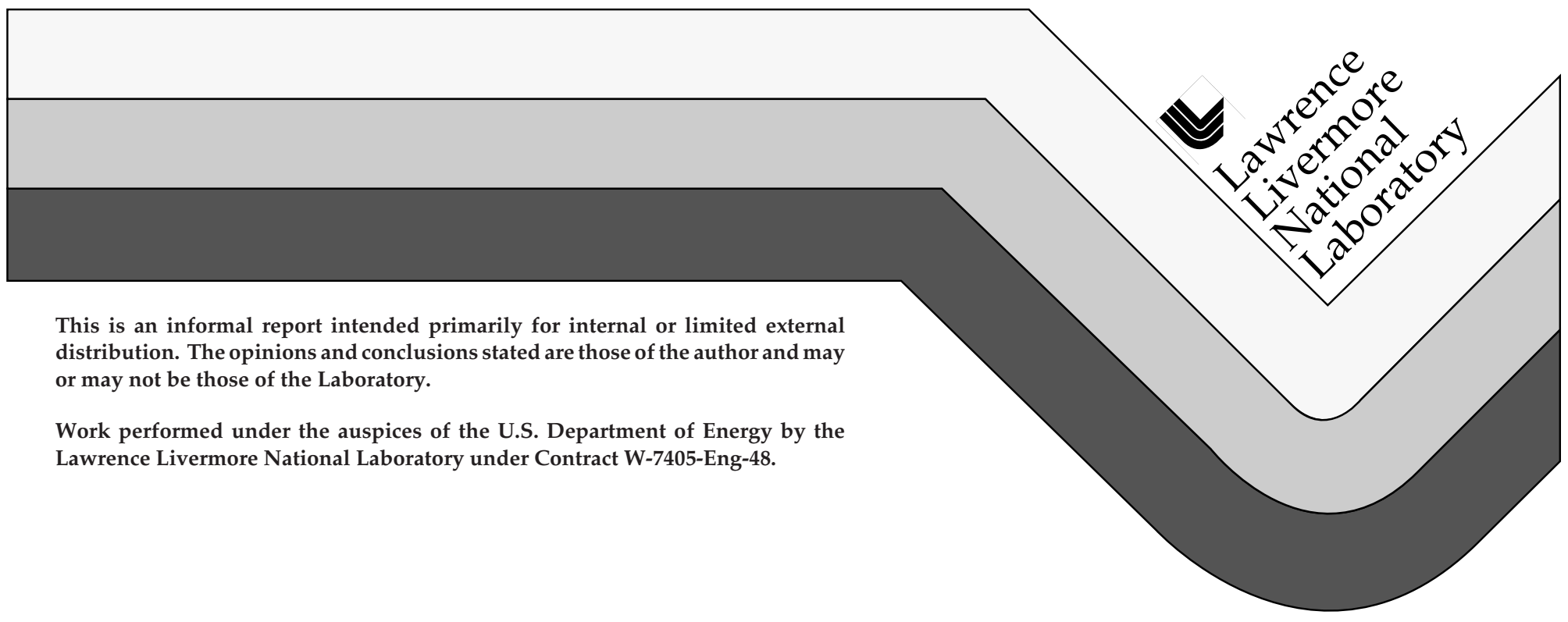




\section{DISCLAIMER}

This document was prepared as an account of work sponsored by an agency of the United States Government. Neither the United States Government nor the University of California nor any of their employees, makes any warranty, express or implied, or assumes any legal liability or responsibility for the accuracy, completeness, or usefulness of any information, apparatus, product, or process disclosed, or represents that its use would not infringe privately owned rights. Reference herein to any specific commercial product, process, or service by trade name, trademark, manufacturer, or otherwise, does not necessarily constitute or imply its endorsement, recommendation, or favoring by the United States Government or the University of California. The views and opinions of authors expressed herein do not necessarily state or reflect those of the United States Government or the University of California, and shall not be used for advertising or product endorsement purposes.

This report has been reproduced directly from the best available copy.

Available to DOE and DOE contractors from the Office of Scientific and Technical Information P.O. Box 62, Oak Ridge, TN 37831

Prices available from (423) 576-8401

Available to the public from the National Technical Information Service

U.S. Department of Commerce 5285 Port Royal Rd. Springfield, VA 22161 


\title{
CODE DEVELOPMENT FOR OPACITY DATABASE
}

\author{
Balazs Rozsnyai \\ A- Division
}

\section{July 1998}

\begin{abstract}
Calculations are presented for copper and beryllium as an example for the proposed new opacity database.
\end{abstract}

\section{Introduction.}

In connection with Stockpile Stewardship and designing meaningful experiments for the forthcoming NIF laser, the need of establishing a useful opacity database for single elements has become acute. An up-to-date opacity database has several requirements; it has to provide the best state of the art opacities and also it has to be in such a format that it lends itself to an easy calculation of mixture opacities. Ir view of the anticipated large scale calculations, the second requirement is absolutely essential. After some lengthy considerations the decision was made to establish a new single element opacity database at LLNL. This database will have the temperature and the averaged free electron density as an input grid. The underlying physical thought behind the idea of using the free electron density as an input variable is that unde that condition the mixing of single element components is dynamically correct. We investigate the above assertion by presenting calculations for cooper and beryllium. Cooper and beryllium present an interesting study case because a copper-beryllium mixture is an alternate to the brominated plastic in NIF ICF capsule designs. In Section II we present some rudimentary theoretical formulas and in Section III we analyze computational results, as given by the new HOPE opacity code.

\section{Theoretical Considerations.}

In this section we present the fundamentals in connection with the free electron density. Details of the quantum mechanics of hot ionized plasmas and opacity were presented elsewhere ${ }^{1,2}$ For details the reader is referred to Refs. 1 and 2 and to references given there. The averaged free electron density and the Fermi level associated with it are given by

$$
\mathrm{n}_{\mathrm{e}}=\mathrm{n}_{\mathrm{i}} \angle^{\pi}=\frac{4 \pi}{h^{3}}(2 \mathrm{mkT})^{3 / 2} \mathrm{~F}_{1 / 2}\left(\mu_{\mathrm{fr}} / \mathrm{kT}\right)
$$

where $n_{i}$ is the ion density and $\mu_{\mathrm{fr}}$ refers to the Fermi level associated with the free electron density and it is not the same as the Fermi level $\mu$ for the combined self - consistent bound and free electron system, which has more physical meaning. The electron pressure associated with Eq.(II 1) is

$$
\mathrm{P}={ }_{3}^{2} \mathrm{k} \operatorname{T\eta }_{\mathrm{e}} \frac{\mathrm{r}_{3 / 2}(\eta)}{\mathrm{F}_{1 / 2}(\eta)}
$$




$$
\text { where } \eta=\mu_{\mathrm{fr}} / \mathrm{kT}
$$

and the F-s stand for the usual Fermi-Dirac integrals. The cardinal point of the $n_{e}$ input is to find $n_{i}$ and $Z^{*} v i$ $\mathrm{Eq}(\mathrm{II} 1)$, and which are not known a priori. This procedure may vary from code to code and for a self consistent Hartree-Slater code it involves a number of iterations. Once this is done the pressure given by Eq.(II 2) is fixed. However, that pressure is fictitious because it assumes a free, non-interacting electron gas. When for two different elements for a given $n_{e}$ the "true" electron pressures agree, then $n_{e}$ is a good input $f_{1}$ mixing. The precise meaning of a "true" electron pressure are is debatable. Below, we try to give a simple discussion. First, we note that the "true" free electron density is a position dependent quantity, which in the Thomas-Fermi approximation is given by

$$
\rho_{f}(r)=\frac{4 \pi}{h^{3}}(2 m k T)^{3 / 2} I_{1 / 2}[\eta(r)]
$$

where $\quad \eta(r)=\frac{\mu-V(r)}{k T}, V(r)$ is the self-consistent potential and $\mu$ is the "true" self-consistent Fermilevel, applied to both bound and free electrons, and $I_{1 / 2}$ stands for an incomplete Fermi-Dirac integral. $Z^{*}$ and $\rho_{\mathrm{f}}(\mathrm{r})$ are related by

$$
4 \pi \rho_{0}^{r_{0}} \rho_{f}(r) r^{2} d r=Z^{*}
$$

In Eq.(II 4) $r_{0}$ stands for the ion-sphere radius whose magnitude is determined by the ion density. The "true" electron pressure, or at least the one which is consistent with the self-consistent Hartree-Slater state it represents, is given by

$$
3 \mathrm{P} \Omega=2 \mathrm{E}_{\mathrm{kin}}-\left\langle\mathrm{r} \frac{\mathrm{V} \mathrm{V}(\mathrm{r})}{f \mathrm{r}}\right\rangle
$$

where $\Omega$ is the volume of the ion-sphere radius and $\mathrm{V}(\mathrm{r})$ is the self-consistent electron potential, including exchange. In fact, Eq (II 5) is a generalization of the virial theorem. In the Thomas-Fermi approximation the pressure is given by

$$
r=\frac{8 \pi}{3 h^{3}}(2 m k T)^{3 / 2} k_{T F}^{+} \frac{\mu-V\left(r_{0}\right)}{k T}
$$

Using the Thomas-Fermi expressions for the kinetic and potential energies, equations (II 6), and (II 5) are connected by a simple integration by parts.

The HOPE code calculates the contribution to the pressure from the bound and free electrons separately. For the free part the Thomas-Fermi approximation is used, whereas for the bound part the self-consistent bound wave functions are used. The contribution to the pressure by the free electrons is analogous to $\mathrm{Eq}(\mathrm{II} 6)$ and is given by

$$
r_{f}=\frac{8 \pi}{3{ }^{2}}(2 m k T)^{3 / 2} k T I_{3 / 2} \frac{\mu-V\left(r_{0}\right)}{k T}
$$

the only difference being the replacement of the Fermi-Dirac integral by the incomplete Fermi-Dirac integral. The quantum-mechanical formula for the pressure due to bound electrons is given by 


$$
3 P_{b} \Omega=\frac{h^{2}}{2 m} p_{b}\left[r\left(R_{b}^{\prime}\right)^{2}-\left(R R^{\prime}\right)_{b}-r\left(R R^{\prime}\right)_{b}\right]_{r=r_{0}}
$$

where $r_{0}$ stands for the ion sphere radius, $R_{b}$ for the radial part of the wave function of a bound state labeled $t$ $\mathrm{b}, \mathrm{p}_{\mathrm{b}}$ for the population of the bound level and the summation goes over the bound states. Equation (II 8) hic negative terms too which in the case of zero temperature give an approximation of the cohesion energy. The applicability of the free electron density as input depends whether or not that for a given $n_{e}$ and kT the self-consistent electron pressures, as given by the sum of Eqs.(II 7) and (II 8), agree for all components in a mixture. If that happens then the code is consistent and $n_{e}$ as an input is appropriate for mixture calculations. Still remains the issue of code compatibility. Two codes are compatible if for a given element, $\mathrm{n}_{\mathrm{e}}$ and $\mathrm{kT}$ they predict the same density in terms of $\mathrm{g} / \mathrm{cc}$. In that case mixture calculations can be performed by computing th different elements with different codes which are compatible with each other.

In the next Section we present some computational results for copper and beryllium.

\section{Computations.}

For a study case we computed an opacity table for copper $(\mathrm{Z}=29)$ and beryllium $(\mathrm{Z}=4)$ in the range of $\mathrm{kT}=10-1.6 \mathrm{x} 10^{4} \mathrm{eV}$ and $\mathrm{n}_{\mathrm{e}}=10^{19}-10^{27}\left(\mathrm{~cm}^{-3}\right)$. Although the objective is to produce opacity tables, in this report we focus on the associated EOS values of pressure and density. For each pair of input variables $n_{e}$ an kT, the HOPE code goes through a set of iterations until the "true" self-consistent position dependent free electron density, when averaged, reproduces the input $\mathrm{n}_{\mathrm{e}}$ as given by Eqs(II. 1) and (II. 4). In the calculations presented here the requirements were set up so that the input $n_{e}-s$ were reproduced within $1 \%$ accuracy. Figure 1 shows examples of electron pressures as given by Eqs.(II 7) and (II 8) in the self-consistent fields (SCF) of beryllium and cooper. We present the curves in the low temperature region where the physics details of EOS are important. Ideally, for proper dynamic mixing the beryllium and copper pressures should be equal, which is not exactly the case, as shown in Fig. 2, where we show the respective ratios. We can see that in the region $\mathrm{n}_{\mathrm{e}}=5 \times 10^{25}$ there is a dip in the Be/Cu SCF pressure ratios, which still has to be investigatec Since the averaged densities $n_{e}$ are equal, the electron pressures given by Eq(II 2), the "non-interacting" (NI) electron gas formula, have to be equal 'a fortiori', as shown for kT=10 eV in Fig. 3. They are not exactly equal to the extent of numerical noise, as shown in Fig. 4 for kT=10 and $100 \mathrm{eV}$. The comparison of the SC and NI pressures are given in Fig. 5. The SCF pressures are invariably below the NI pressures, due to the attractive field of the central ion. Next, on Figs. 6 and 7 for a selected few isotherms we show the Rosseland mean opacities of beryllium and copper, respectively.

For the free-free absorption or inverse bremsstrahlung we calculated the free-free Gaunt factor using the formula ${ }^{3}$

$$
g^{f f}\left(k_{i} k_{f}\right)=\frac{\sqrt{3}}{\pi\left(Z^{*}\right)^{2}} k_{i} k_{f}{ }_{I=0}^{\times}(l+1) \sin ^{2}\left[\delta_{I}(k)-\delta_{I+1}(k)\right]
$$

where $\delta$ stands for the phase shift, the wave vectors are give in units of inverse Bohr radius and $\mathrm{k}$ is the average wave vector between the initial and final continuum state. The author guesses that the usage of Eq(II 1) may be responsible for the dips in the beryllium Rosseland means, but that needs a closer investigation. Finally, on Figs. 8 and 9 we present some computed opacities for a beryllium copper mixture. We took the mixture of $99 \%$ beryllium and 1\% copper, which was a study case at the WorkOp-IV:97 opacity workshop ${ }^{4}$ and it is an alternate to brominated plastic in NIF ICF capsule designs. We investigate two cases, $\mathrm{kT}=64 \mathrm{eV}$ and $\mathrm{n}_{\mathrm{e}}=10^{24} \mathrm{~cm}^{-3}$, and $\mathrm{kT}=32 \mathrm{eV}$ and $\mathrm{n}_{\mathrm{e}}=10^{26} \mathrm{~cm}^{-3}$. The second case is in the region where the $\mathrm{SCF} B e / \mathrm{Cu}$ electron pressure ratios are the worse. For each case we present two sets of frequency dependent opacities, or which was obtained from the beryllium and copper opacity tables and the second which was calculated 
directly by equating the Thomas-Fermi values of the electron Fermi levels for the beryllium and copper components, and proceeding to make full opacity calculations

for both elements. The second version of mixture calculation are shown by the dashed curves, it is time consuming and it was used in the past at LLNL. The first version, using the tables, takes a few seconds to complete. In both cases there seems to be a slight difference between the K and L shells, but the Rosseland mean opacities are practically identical.

\section{Conclusion.}

It seems that using the averaged free electron density and temperature as a grid for developing a single element opacity database is an appropriate choice for facilitating the computation of mixture opacities. Once the single element database is established, the computation of mixture opacities is a simple matter. We still have to investigate the subject of the compatibility of the HOPE, OPAL and STA codes.

\section{Acknowledgment.}

This work was performed under the auspices of the U.S. Department of Energy by Lawrence Livermore National Laboratory under Contract No. W-7405-Eng-48. 


\section{References:}

1. B. F. Rozsnyai, Phys. Rev. A5, 1137 (1972).

2. B. F. Rozsnyai, Defense Research Review, 1, 21, (1989).

3. B. F. Rozsnyai and M. Lamoureux, JQSRT 43, 381 (1990).

4. WorkOp-IV:97, Fourth International Opacity Workshop and Code Comparison Study May 26-30, 1997, Madrid, Spain. 


\section{Figure Captions.}

Fig. 1. "Self-consistent"(SCF) electron pressures versus the averaged free electron density $n_{e}$ of beryllium and copper from kT=10 to $200 \mathrm{eV}$. The isotherms I, II, III, and IV contain both elements.

Fig.2. Be/Cu SCF pressure ratios of the isotherms of Fig.1

Fig. 3. Electron pressures of beryllium and copper versus $n_{e}$ at $k T=10 \mathrm{eV}$ as predicted by the "non -interacting (NI) electron gas formula of Eq.(II 2).

Fig. 4. Be/Cu NI electron pressure ratios at $\mathrm{kT}=10$ and $100 \mathrm{eV}$.

Fig. 5. SCF/NI electron pressure ratios for beryllium and copper. The NI pressures are always larger than the SCF pressures, thus the ratios are $<1$.

Fig. 6. Radiative Rosseland mean opacities for beryllium.

Fig. 7. Same as Fig. 6 for copper.

Fig. 8. Comparison of mixture opacities for a beryllium-copper mixture for $\mathrm{kT}=64 \mathrm{eV}, 5.706 \mathrm{~g} / \mathrm{cc}$ density. Full line, using the single element opacity tables, dashed line, direct 'brute force' calculations for the components.

Fig. 9. Same as Fig. 8 for kT=32 eV and $404 \mathrm{~g} / \mathrm{cc}$ density. 
SCF pressures of $\mathrm{Be}$ and $\mathrm{Cu}$ versus ne

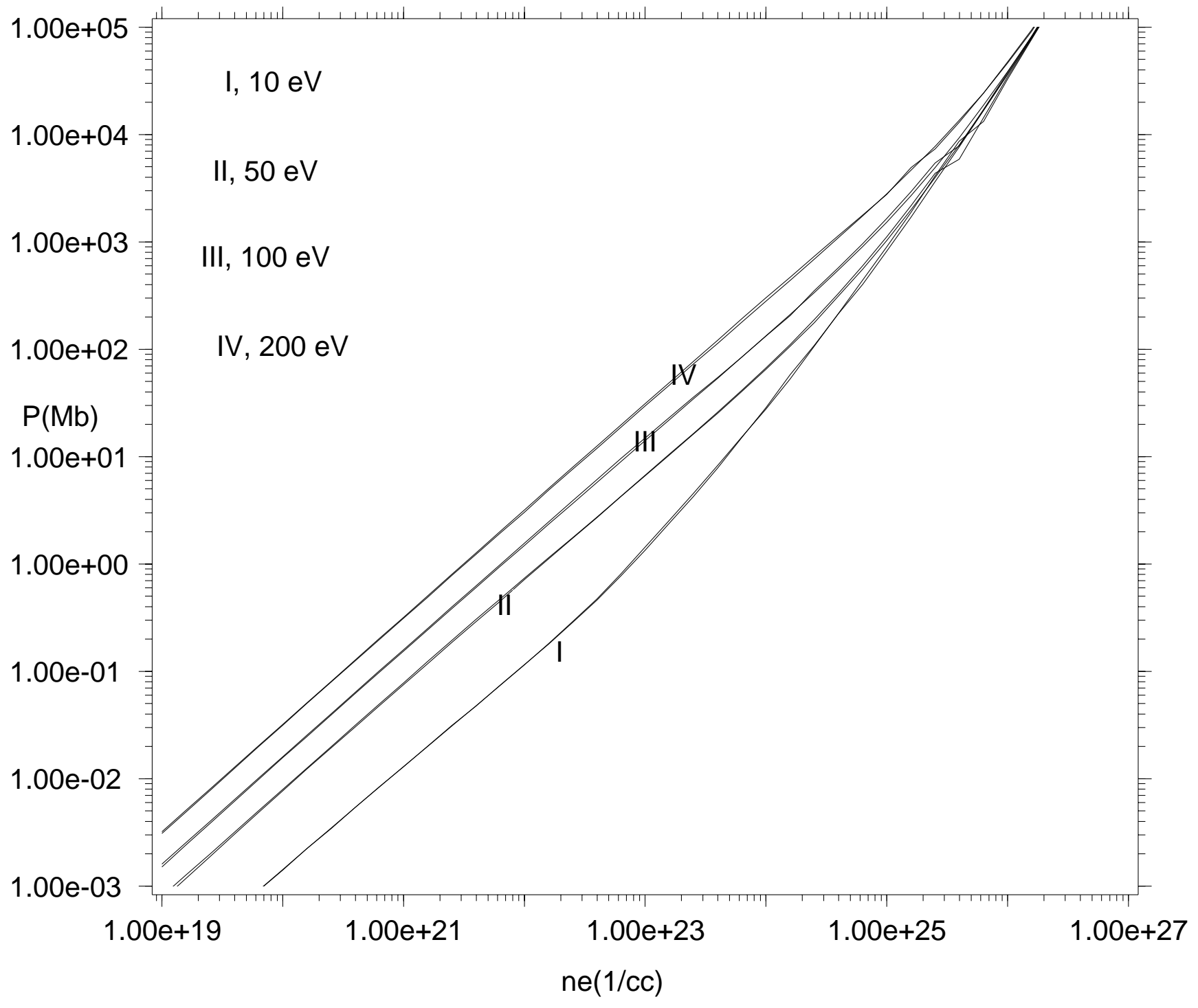




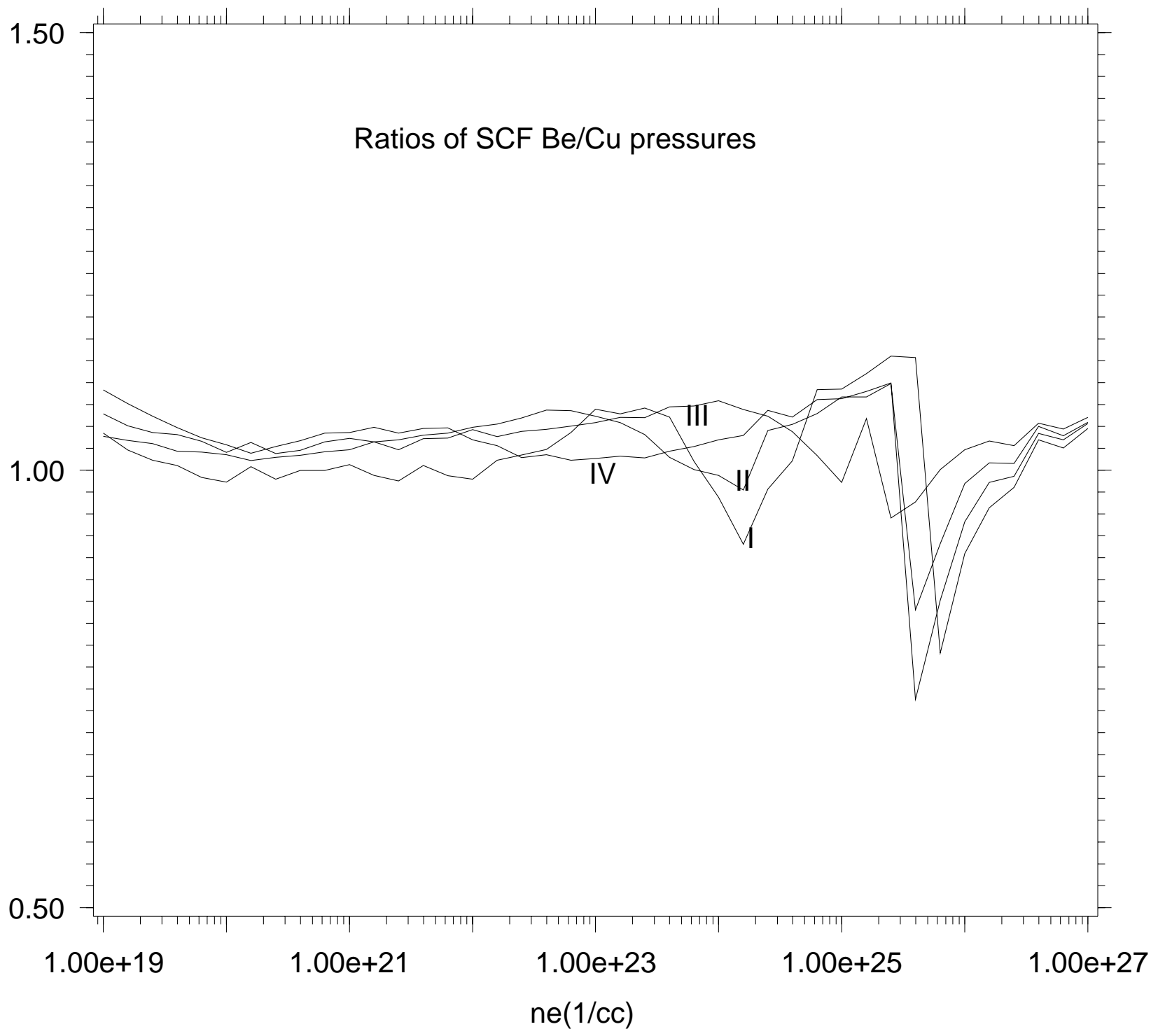




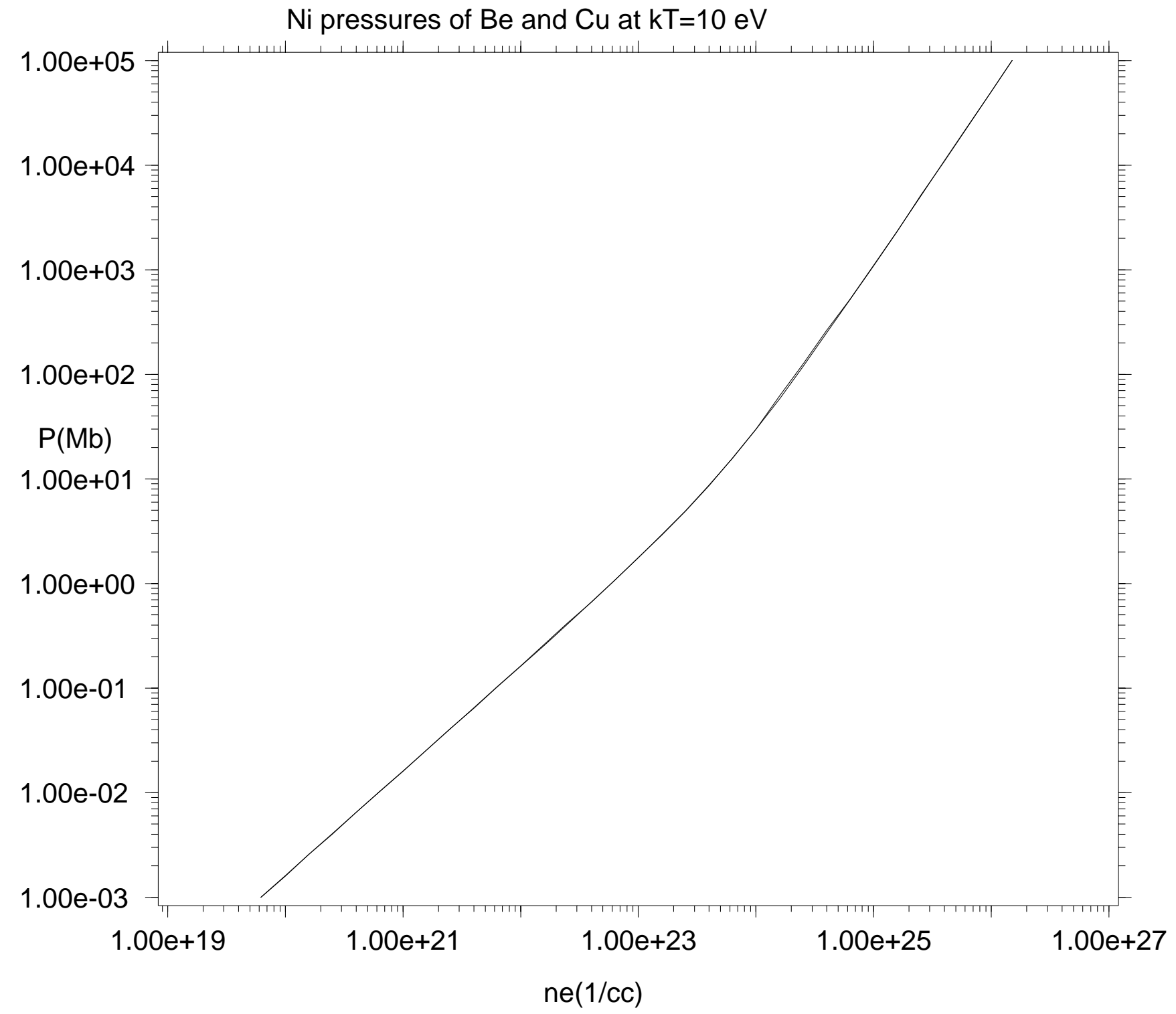




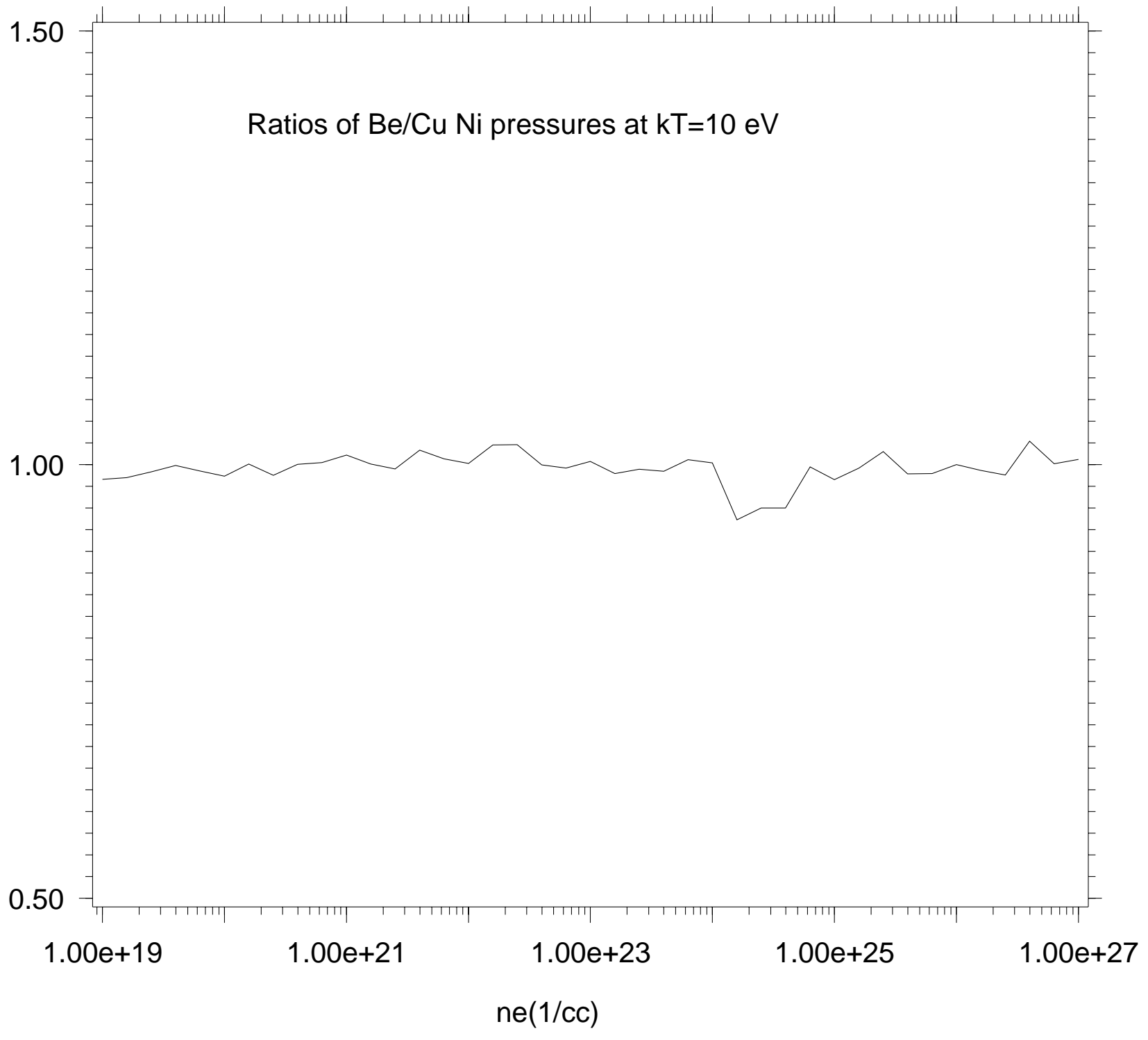


Ratios of SCF/Ni pressures.

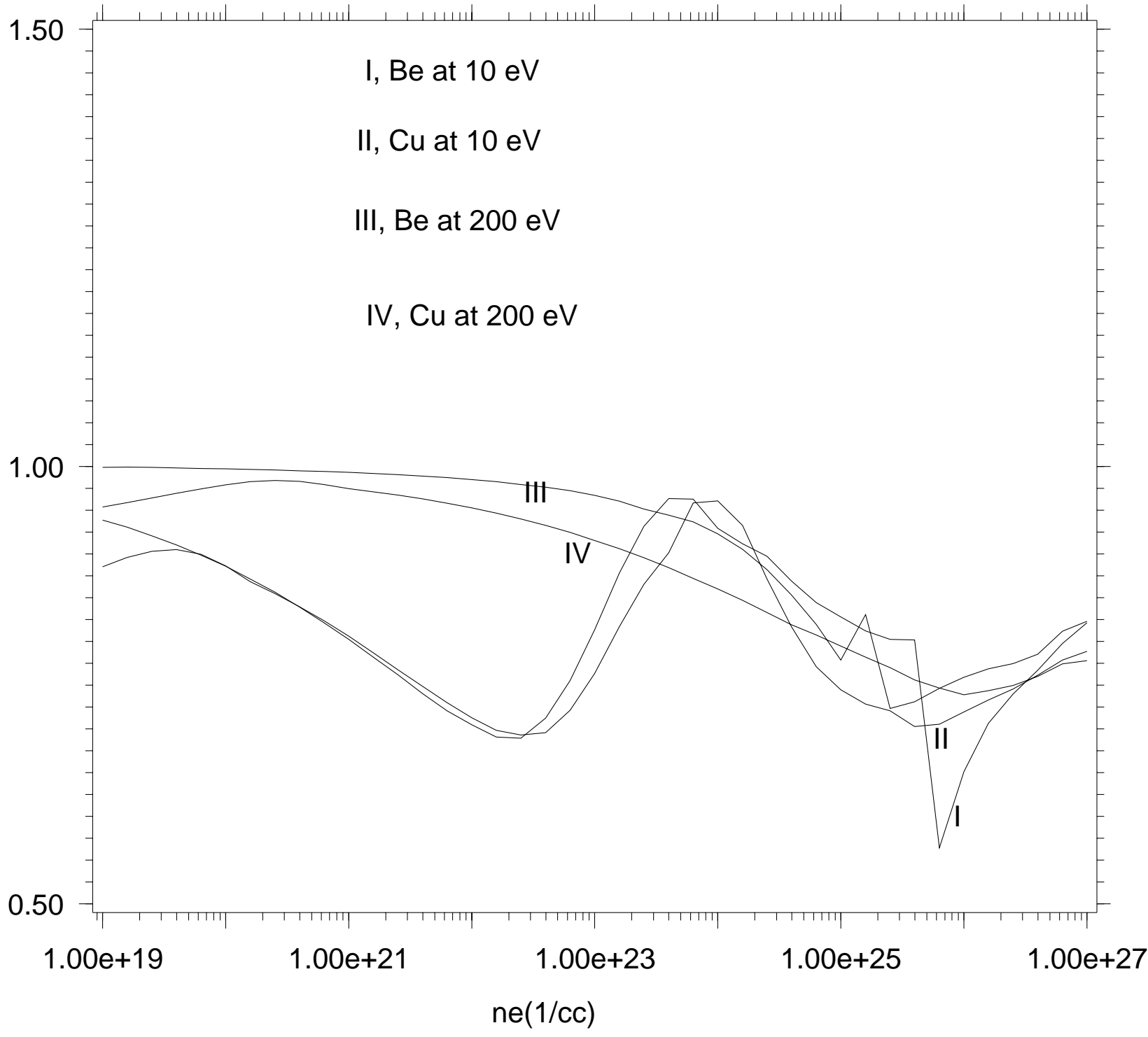


Rosseland mean opacities of $\mathrm{Be}$

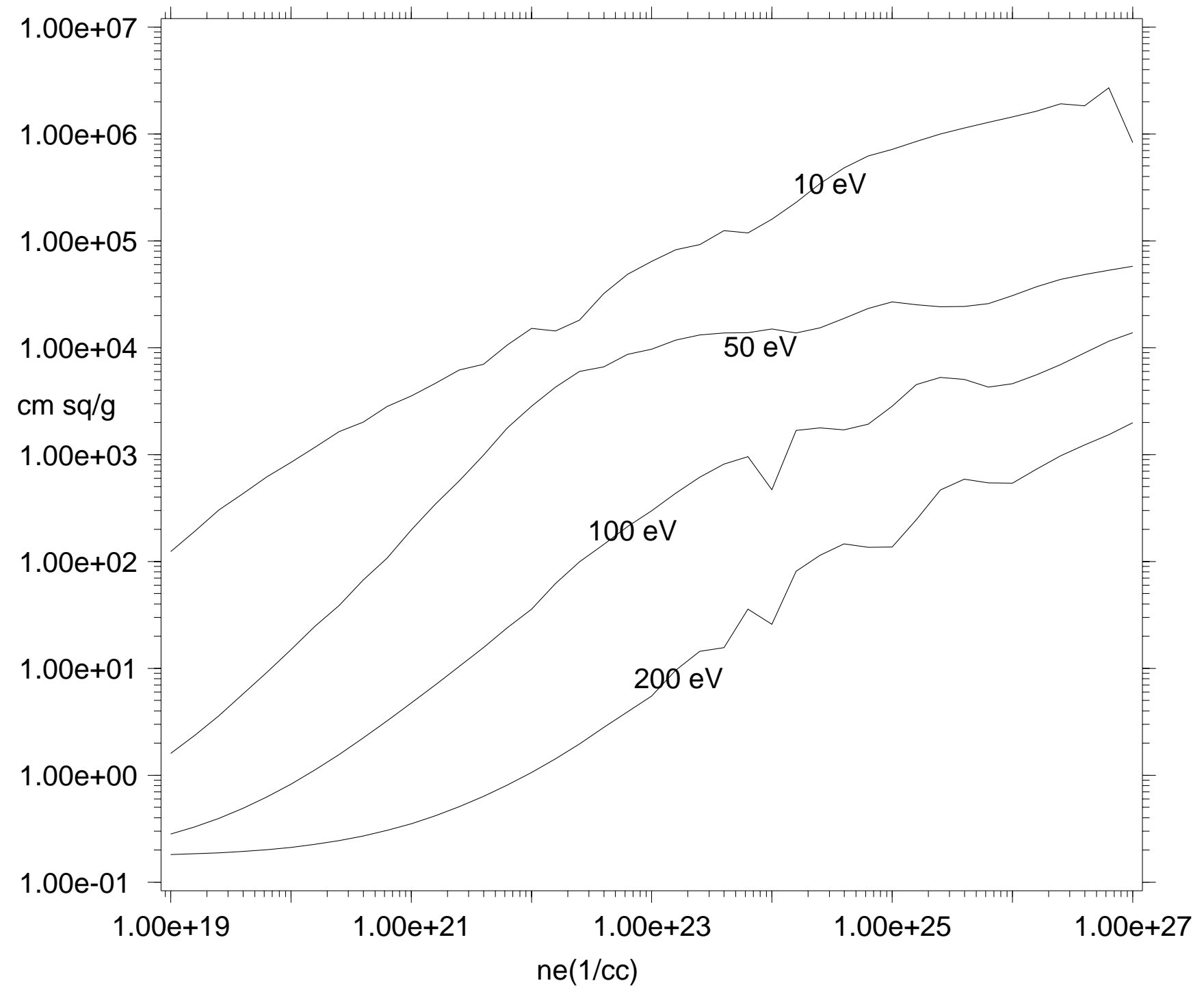




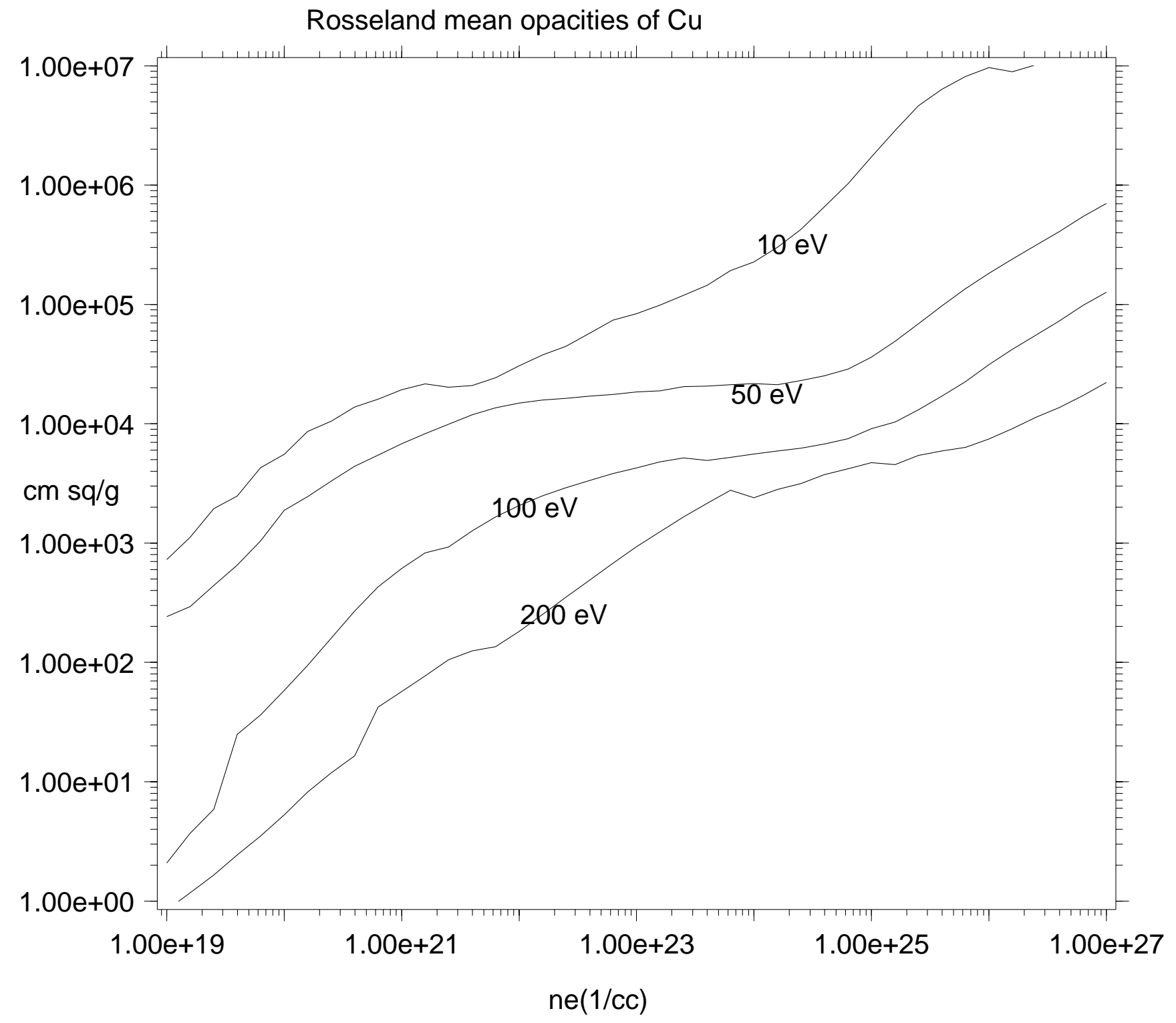




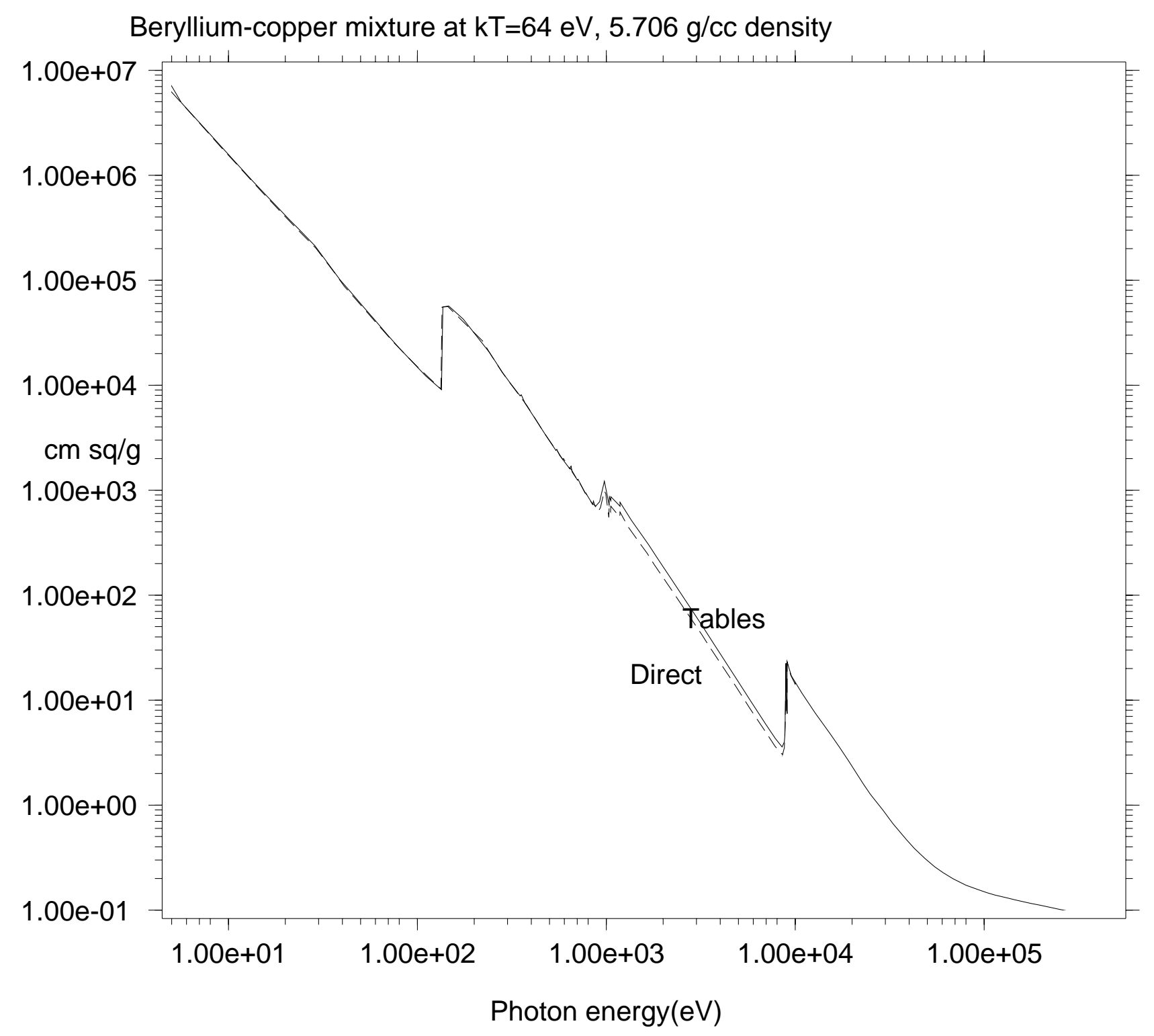


Beryllium-copper mixture at $\mathrm{kT}=32 \mathrm{eV}, 404 . \mathrm{g} / \mathrm{cc}$ density

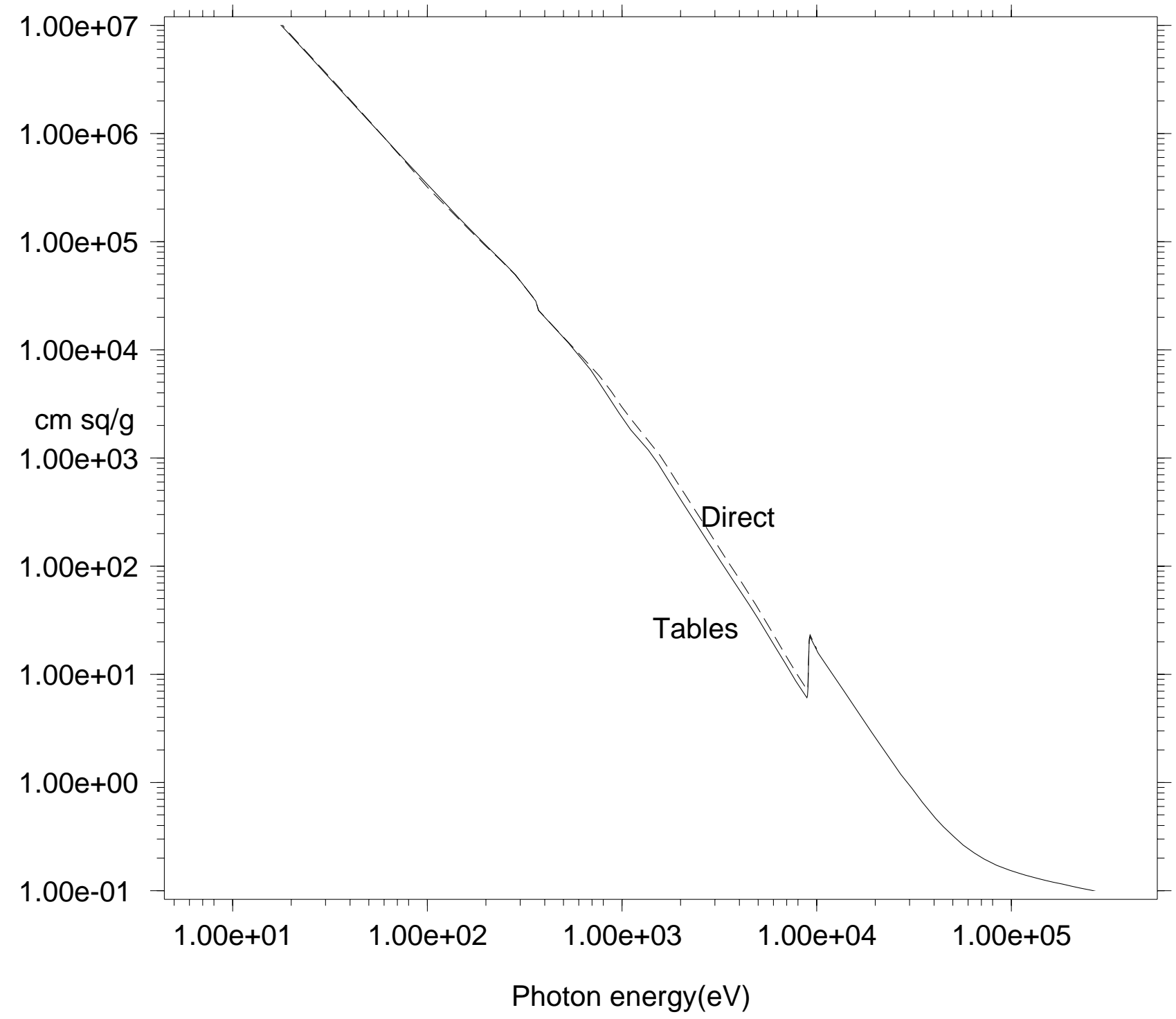




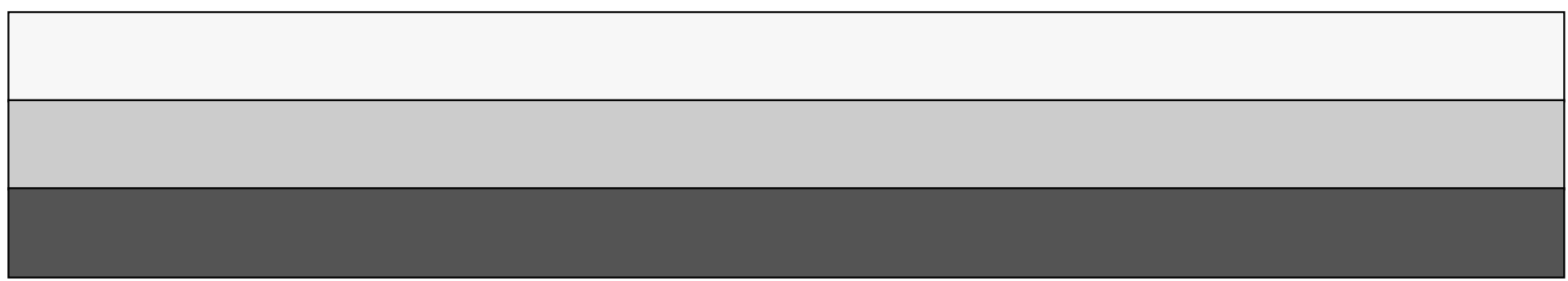

